\title{
Antioxidant and Vascular Effects of Gliclazide in Type 2 Diabetic Rats Fed High-Fat Diet
}

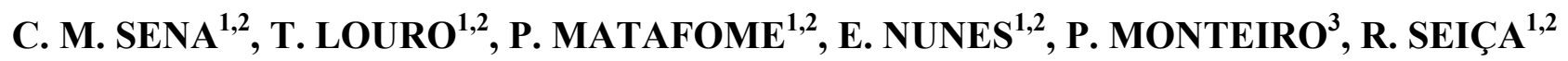 \\ ${ }^{1}$ Institute of Physiology, Faculty of Medicine, University of Coimbra, ${ }^{2}$ IBILI, Faculty of Medicine, \\ University of Coimbra, ${ }^{3}$ Basic Research Unit in Cardiology, Cardiology Department, Coimbra \\ University Hospital and Medical School, Coimbra, Portugal
}

Received January 3, 2008

Accepted February 13, 2008

On-line April 1, 2008

\section{Summary}

Diabetes mellitus is characterized by oxidative stress, which in turn determines endothelial dysfunction. Gliclazide is a sulphonylurea antidiabetic drug with antioxidant effects due to its azabicyclo-octyl ring. It has been reported to potentially protect the vasculature through improvements in plasma lipid levels and platelet function. We hypothesized that gliclazide has a beneficial effect on endothelial function in Goto-Kakizaki rats (GK), an animal model of type 2 diabetes fed an atherogenic diet for 4 months. We evaluated the influence of gliclazide on both metabolic and oxidative status and NO-mediated vasodilation. GKAD rats showed increased oxidative stress and impaired endothelium-dependent vasodilation. GKAD rats treated with gliclazide showed increased sensitivity to NO-mediated vasodilation, a significant decrease in fasting glycemia and insulinemia, and a significant decrease in systemic oxidative stress. In conclusion, our results suggest that gliclazide treatment improves NO-mediated vasodilation in diabetic GK rats with dyslipidemia probably due to its antioxidant effects, although we cannot rule out substantial benefits due to a reduction in fasting blood glucose. The availability of a compound that simultaneously decreases hyperglycemia, hyperinsulinemia, and inhibits oxidative stress is a promising therapeutic candidate for the prevention of vascular complications of diabetes.
\end{abstract}

\section{Key words}

Endothelium • Nitric oxide • Diabetes mellitus • Gliclazide • Oxidative stress

\section{Corresponding author}

C. M. Sena, Institute of Physiology, Sub-unidade 1, Pólo III, Faculty of Medicine, University of Coimbra, Azinhaga de Santa Comba, Celas, 3000-504 Coimbra, Portugal. Fax: +351-239480034. E-mail: csena@ci.uc.pt

\section{Introduction}

The increase in oxidative stress that is associated with the chronic hyperglycemia of diabetes mellitus plays a key role in the genesis of endothelial dysfunction (Giugliano et al. 1996, Pennathur and Heinecke 2004). Agents with antioxidant properties improve the function of the vessels in animal models of diabetes (Da Ros et al. 2004, Hamilton et al. 2007). We have recently reported that impaired endothelial dysfunction and increased oxidative stress was improved by $\alpha$-lipoic acid, in old Goto-Kakizaki diabetic rats (Sena et al. 2008).

Gliclazide is a second-generation sulfonylurea antihyperglycemic agent (Campbell et al. 1991) that stimulates insulin secretion from pancreatic $\beta$ cells by inhibiting ATP-dependent potassium channels. ATPdependent potassium channels also mediate a variety of functions in heart and blood vessels (Nichols and Lederer 1992) Thus it is possible that sulfonylurea agents would have some effects on the vascular function aside from their effects on glycemic control. In diabetic animal models, it has also been reported that gliclazide potentially protects the vasculature through improvements in plasma lipids and platelet function (Palmer and Brogden 1993). Mechanisms may include the ability of the drug to increase tissue plasminogen activator, and its properties as a free radical scavenger. (Scott et al. 1991, Jennings et al. 1992, Desfaits et al. 1997, O'Brien et al. 2000). The aim of the present work was to address whether the gliclazide influenced endothelial dysfunction and oxidative stress in GotoKakizaki diabetic rats fed a high-fat diet. 
Table 1. Influence of gliclazide on body weight, blood glucose, fasting insulin, lipids and free fatty acids (FFAs) in GKAD rats.

\begin{tabular}{lllll}
\hline & WISTAR & GK & GKAD & GKAD+G \\
\hline Body weight $(\mathrm{g})$ & $403.9 \pm 6.9$ & $312.7 \pm 5.8^{* * *}$ & $387.3 \pm 4.3^{\S \S \S}$ & $395.3 \pm 5.2^{\S \S \S}$ \\
FBG (mmol/l) & $3.64 \pm 0.11$ & $5.17 \pm 0.14^{* * *}$ & $7.78 \pm 0.16^{* * *} \S \S \S$ & $5.45 \pm 0.13^{* * * \phi \phi \phi}$ \\
BG 2 h after load (mmol/l) & $4.65 \pm 0.15$ & $16.62 \pm 0.4^{* * *}$ & $16.83 \pm 0.83^{* * *}$ & $17.61 \pm 1.57^{* * *}$ \\
Insulin (pmol/l) & $119.8 \pm 30.1$ & $265.9 \pm 18.6^{* * *}$ & $189.9 \pm 19.1^{* \S}$ & $105.0 \pm 19.2^{\S \S \phi \phi}$ \\
Cholesterol (mmol/l) & $1.82 \pm 0.04$ & $2.3 \pm 0.05$ & $2.6 \pm 0.14^{* * *} \S \S \S$ & $2.4 \pm 0.06^{* * *} \phi$ \\
Non-HDL cholesterol (mmol/) & $0.7 \pm 0.04$ & $0.71 \pm 0.07$ & $1.29 \pm 0.13^{* * *} \S \S \S$ & $1.18 \pm 0.05^{* * * \phi}$ \\
Triglycerides (mmol/l) & $1.23 \pm 0.09$ & $1.69 \pm 0.13^{*}$ & $2.52 \pm 0.23^{* * *} \S \S \S$ & $2.24 \pm 0.08^{* * *} \S \S$ \\
FFAs (mmol/l) & $0.71 \pm 0.08$ & $0.79 \pm 0.04$ & $1.13 \pm 0.04^{* * *} \S \S$ & $0.66 \pm 0.09^{\phi \phi \phi}$ \\
\hline
\end{tabular}

Values are mean \pm S.E.M. * $\mathrm{P}<0.05, * * * \mathrm{P}<0.001$ vs. Wistar rats; $\S \mathrm{P}<0.05, \S \S \mathrm{P}<0.01, \S \S \S \mathrm{P}<0.001$ vs GK rats; $\phi \mathrm{P}<0.05$, $\phi \phi \phi P<0.001$ vs. GKAD rats. FBG - fasting blood glucose.

\section{Methods}

\section{Experimental animals}

Wistar and Goto-Kakizaki rats were obtained from our local breeding colony (Animal Research Center Laboratory, University Hospital, Coimbra, Portugal). Animals were subjected to a constant daily cycle of $12 \mathrm{~h}$ light and $12 \mathrm{~h}$ darkness and constant temperature $\left(22-24{ }^{\circ} \mathrm{C}\right)$ and humidity (50-60\%), with free access to water and to normal or high-fat diet between 2 and 6 months of age [Special diet 0125 (Modified A04 from Charles River), SAFE, France].

Rats were divided in four experimental groups accordingly [Wistar non-diabetic rats $(\mathrm{W}, \mathrm{n}=12)$, GK diabetic rats fed a normal diet (GK control, $n=16$ ), GK diabetic rats fed an $\mathrm{AD}$ diet (GKAD, $\mathrm{n}=15)$, and GK rats fed an $\mathrm{AD}$ diet and treated with gliclazide $(\mathrm{GKAD}+\mathrm{G}$, $\mathrm{n}=14)]$. Gliclazide (10 mg/kg/day) was administered in drinking water during four weeks before sacrifice. The experimental protocol was approved by the local Institutional Animal Care and Use Committee.

Determination of metabolic and oxidative stress parameters

After a 15 -h fast, animals were anesthetized with ketamine/chlorpromazine. Blood was taken by heart puncture for determination of lipids, lipid peroxides, free fatty acid levels and insulin. For glucose tolerance tests, rats were fasted overnight and were given an intraperitoneal injection of glucose $\left(1.75 \mathrm{~g} \mathrm{~kg}^{-1}\right.$ body weight) in phosphate buffered saline (PBS). Blood glucose was determined by sampling from the tail vein at 0, 30, 60, 90 and 120 min after injection by a glucoseoxidase method using a glucometer (Glucometer-Elite-
Bayer, Portugal S.A.) and compatible reactive test strips. Fasting plasma lipids (total and HDL cholesterol, triglycerides and phospholipids) and plasma insulin levels were quantified using commercially available kits and by an in-house enzyme-linked immunosorbent assay (Seiça et al. 2004, Sena et al. 2007), respectively. Plasma free fatty acids (FFA) levels were evaluated using enzymatic assay kits (Roche Applied Science, Portugal). Rats were placed in metabolic cages for $24 \mathrm{~h}$ and urine collected. Urinary 8-hydroxydeoxyguanosine (8-OHdG), and plasma carbonyl protein concentration were measured using ELISA kits (OXIS health Products, Portland, OR, USA, Cayman Chemical Company, USA).

\section{Isometric tension studies}

Aorta were rapidly excised and freed of connective tissue. The aorta was divided into two segments (4 mm width). Ring segments were mounted between stainless steel triangles into individual organ chambers filled with oxygenated $\left(95 \% \mathrm{O}_{2}, 5 \% \mathrm{CO}_{2}\right)$ KrebsHenseleit buffer $\left(37^{\circ} \mathrm{C}\right.$, pH 7.4) (composition in mmol $1^{-1}$ : $\mathrm{NaCl} 119 ; \mathrm{KCl} 4.7 ; \mathrm{CaCl}_{2}$ 1.6; $\mathrm{MgSO}_{4}$ 1.2; $\mathrm{NaHCO}_{3} 25$; $\mathrm{KH}_{2} \mathrm{PO}_{4}$ 1.2; glucose 11.0). Indomethacin in a concentration of $10 \mu \mathrm{mol} \mathrm{l}^{-1}$ was present in all the experiments to inhibit prostaglandin synthesis. Aortic rings were subjected to a resting tension of $1.5 \mathrm{~g}$. After equilibration for $60 \mathrm{~min}$ all vessels were preconstricted with $0.3 \mu \mathrm{M}$ phenylephrine. Ligand-stimulated receptormediated NO bioavailability was assessed by a dosedependent relaxation to acetylcholine (ACh, $10^{-8}$ to $10^{-3}$ $\mathrm{M}$ ), whereas sodium nitroprusside (SNP, $10^{-8}$ to $10^{-3} \mathrm{M}$ ) was used as an endothelium-independent agonist. Relaxation responses to ACh and SNP were expressed as percentage of relaxation from submaximal phenylephrine- 

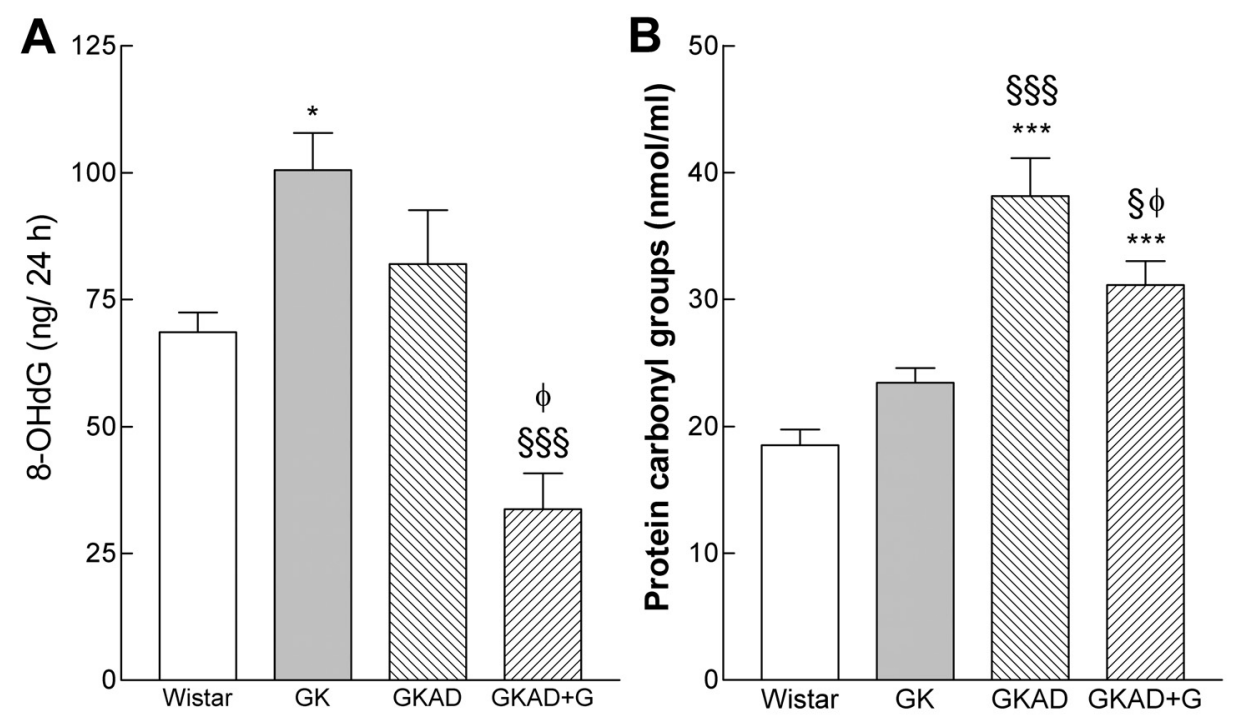

Fig. 1. Reduction of oxidative stress parameters by gliclazide. Panels show urinary 8-OHdG (A) and plasma protein-bound carbonyls (B) levels in Wistar, GK control and GK rats fed a high-fat diet with $(G K A D+G)$ or without gliclazide (GKAD). Results are mean \pm SEM. *** $\mathrm{P}<0.001$ vs. Wistar group; ${ }^{\S} \mathrm{P}<0.05,{ }^{\S \S \S} \mathrm{P}<0.001$ vs. GK group; $\phi P<0.05$ vs. GKAD group.
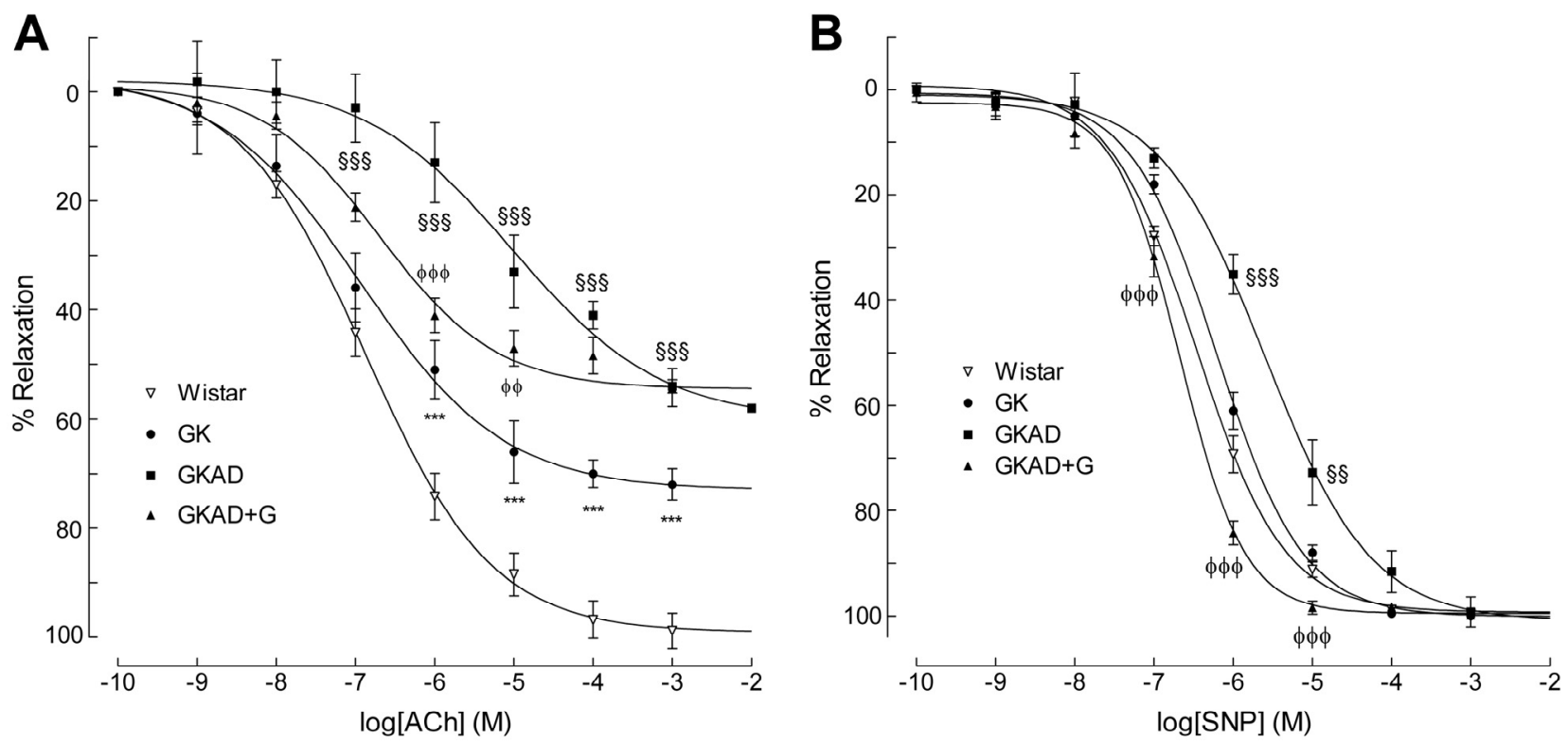

Fig. 2. Effects of diabetes, high-fat diet and gliclazide treatment on vasodilatory responses to acetylcholine (A) and sodium nitroprusside (B) of phenylephrine-precontracted rat thoracic aortic rings. Vasorelaxation was measured using an isometric force displacement transducer. Data are expressed as mean $\pm \mathrm{SEM}$. *** $\mathrm{P}<0.001$ vs. Wistar group; ${ }^{\S \S} \mathrm{P}<0.01, \S \S \S \mathrm{P}<0.001$ vs. GK group; $\phi \phi \mathrm{P}<0.01, \phi \phi \phi \mathrm{P}<0.001$ vs. GKAD group.

induced constriction $\left(10^{-7} \mathrm{M}\right)$. A cumulative dose-efficacy curve was determined. Regression analysis using three data points along the linear section of the concentrationresponse curve was applied to generate an equation from which the $\mathrm{EC}_{50}$ values were determined (Sena et al. 2008).

\section{Statistical analysis}

All data were analyzed by standard computer programs (GraphPad Prism PC Software version 3.0, ANOVA) and are expressed as mean \pm S.E.M. Significant differences were evaluated using either the $t$-test or ANOVA. $\mathrm{P}<0.05$ was considered significant. Dose response curves were fitted by nonlinear regression with simplex algorithm. Relaxation responses were given as the percentage of phenylephrine-induced constriction. Comparisons of dose-response curves were evaluated by 2-way ANOVA for repeated measures.

\section{Results}

Blood parameters and body weight

Diabetic GK rats fed a normal chow diet were 


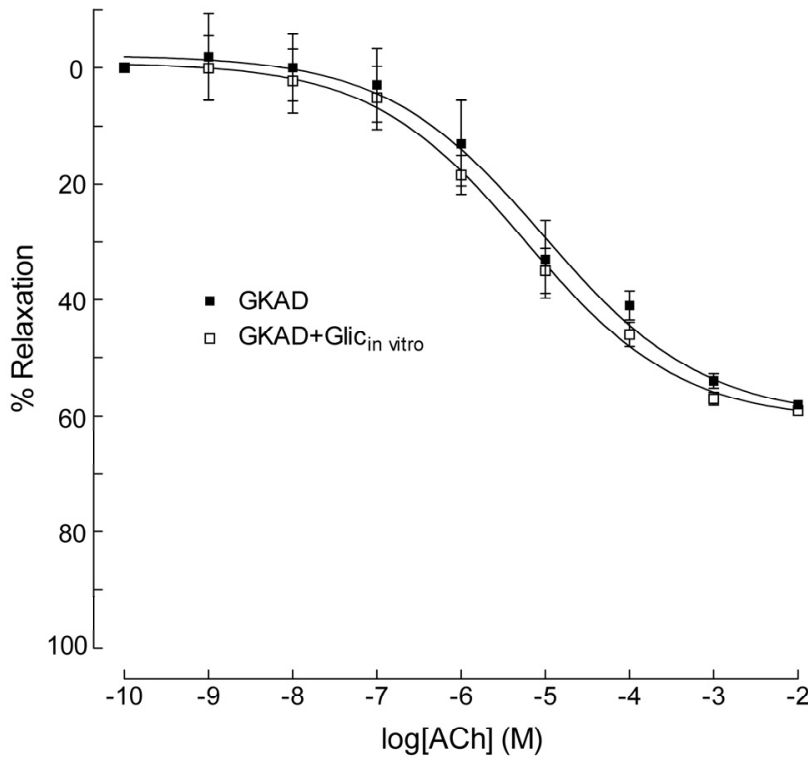

Fig. 3. Acetylcholine-induced relaxations of phenylephrineprecontracted rat thoracic aortic rings from diabetic GK rats fed a high-fat diet (GKAD). In vitro addition of gliclazide $(10 \mu \mathrm{M})$ to rings from GKAD rats had no significant effect.

hyperglycemic and hyperinsulinemic compared to Wistar rats. GK rats were normolipidemic and their body weight was decreased when compared with Wistar rats (Table 1).

After four months of high-fat diet feeding, GK diabetic rats showed a significant increase in body weight, fasting glycemia, total and non-HDL-cholesterol, triglycerides and FFA levels when compared with GK rats receiving a standard diet (Table 1). Treatment of the animals with gliclazide in drinking water significantly decreased fasting glycemia and insulinemia, total and non-HDL-cholesterol and FFA levels. The treatment did not modify body weight, triglyceride and glucose levels $2 \mathrm{~h}$ after glucose load (Table 1). Food consumption and water intake did not significantly change over the experimental period between the different groups studied (data not shown).

\section{Oxidative stress biomarkers}

Urinary levels of $8-\mathrm{OHdG}$ were significantly higher in GK rats, when compared to age-matched Wistar rats (Fig. 1A), while plasma levels of protein carbonyl compounds were not statistically different between the two groups. Feeding AD diet for 4 months to GK rats significantly increased protein carbonyl compounds levels by $62 \%$ (Figs $1 \mathrm{~A}$ and $1 \mathrm{~B}$ ) and did not changed the levels of 8-OHdG. Supplementation with gliclazide significantly reduced these oxidative stress parameters (Figs 1A and 1B).
ACh- and NO-induced relaxation in rat aortic segments

The addition of ACh or NO to vessels previously contracted with phenylephrine caused a concentrationdependent relaxation (Figs 2A and 2B). In GK rats endothelium-mediated vascular relaxation by $\mathrm{ACh}$ of aortic rings that were previously contracted with phenylephrine was impaired compared with age-matched Wistar rats. Indeed, maximal endothelium-mediated relaxation of phenylephrine-precontracted rings in response to $\mathrm{ACh}$ was decreased by $23 \%$ and the $\mathrm{EC}_{50}$ value of endothelium-independent relaxation to SNP was significantly increased (Figs $2 \mathrm{~A}$ and $2 \mathrm{~B}$ ). The $\mathrm{AD}$ diet further impaired vascular relaxation in response to both $\mathrm{ACh}$ and SNP in GK rats. Gliclazide treatment improved endothelium-dependent vascular relaxation in GK rats fed $\mathrm{AD}$ diet. Indeed, when ACh-induced relaxation was observed in aortic segments from $\mathrm{GKAD}+\mathrm{G}$ a significant increase in sensitivity to ACh was observed without major changes in maximum relaxation (Fig. 2, Table 2, $\mathrm{P}<0.001$ ). Endothelium-denuded rings from all groups showed no relaxation to ACh (data not shown). The addition of gliclazide $(10 \mu \mathrm{mol} / \mathrm{l})$ in vitro to aorta from GKAD rats had no significant effect on relaxation in response to acetylcholine $\left(10^{-9}-10^{-3} \mathrm{~mol} / \mathrm{l}\right.$, Fig. 3).

The $\mathrm{EC}_{50}$ values for $\mathrm{ACh}-$ and NO-evoked relaxation in aortic segments from Wistar and GK diabetic rats are shown in Table 2. The $\mathrm{EC}_{50}$ for $\mathrm{ACh}$ in the GKAD group was significantly increased $(\mathrm{P}<0.001$ vs. untreated diabetic rats). The sensitivity of the response evoked by $\mathrm{ACh}$ or $\mathrm{NO}$ in aortic segments from GKAD $+\mathrm{G}$ was significantly higher than in untreated diabetic animals (Table 2, $\mathrm{EC}_{50}$ values significantly decreased, $\mathrm{P}<0.001)$.

Preincubation of the arterial rings with the NOS inhibitor $\mathrm{N}^{\boldsymbol{w}}$-nitro-L-arginine methyl ester (L-NAME) and the cyclooxygenase inhibitor indomethacin caused residual relaxations of $15 \%$ in response to $\mathrm{ACh}$ in GK rats and Wistar rats (data not shown). These relaxations were not significantly different between the groups studied.

\section{Discussion}

Several studies have demonstrated impairment of endothelium-dependent relaxation associated with diabetes (Fortes et al. 1983, Durante et al. 1988). In GK diabetic rats, the reduction in endothelium-dependent vasodilation has been observed both in experiments with isolated aortic rings (Sena et al. 2008), and mesenteric 
Table 2. Effects of diabetes, high-fat and gliclazide treatment on maximal relaxation responses (\%) and $\mathrm{EC}_{50}$ values in response to acetylcholine and sodium nitroprusside of phenylephrine-precontracted rat thoracic aortic rings.

\begin{tabular}{lllll}
\hline & Wistar & \multicolumn{1}{c}{ GK } & \multicolumn{1}{c}{ GKAD } & \multicolumn{1}{c}{ GKAD+G } \\
\hline Acetylcholine & & & & \\
$\quad \begin{array}{l}\text { EC } 50 \\
\text { Maximal relaxation (\%) }\end{array}$ & $99.1 \pm 0.9$ & $72.9 \pm 1.7^{* * *}$ & $5.02 \pm 0.14^{* * *} \S \S \S$ & $6.69 \pm 0.16^{\phi \phi \phi}$ \\
Sodium nitroprusside & & & $60.2 \pm 2.1^{* * * \S \S}$ & $55.4 \pm 5.4^{* * *} \S \S$ \\
$\quad$ EC $C_{50}$ & $6.47 \pm 0.04$ & $6.2 \pm 0.04^{* * *}$ & $5.6 \pm 0.04^{* * *} \S \S$ & $6.67 \pm 0.03^{* * *} \S \S \S \phi \phi$ \\
Maximal relaxation (\%) & $99.3 \pm 1.3$ & $99.9 \pm 1.3$ & $99.1 \pm 1.6$ & $99.5 \pm 1.1$ \\
\hline
\end{tabular}

Values are mean \pm S.E.M. $E C_{50}$ values are presented as the negative logarithm $\left(-\log _{10} \mathrm{EC}_{50}\right)$ of concentration of the agonist. $* * * \mathrm{P}<0.001$ vs. Wistar rats; ${ }^{\S} \mathrm{P}<0.01,{ }^{\S \S \S} \mathrm{P}<0.001$ vs. GK rats; $\phi \phi \phi \mathrm{P}<0.001$ vs. GKAD rats.

microvessels (Cheng et al. 2001), indicating that diabetic endothelial dysfunction affects both conductance and resistance vessels. In the present work, the results obtained in aortic rings showed impairment in endothelium-mediated responses over the 6-month period of the evolution of diabetes in the GK rat model. High-fat diet significantly impaired endothelial dysfunction and this was improved by treatment with gliclazide for 4 weeks.

It is widely accepted that increased oxidative stress occurs in diabetes (Baynes 1991, Ceriello et al. 1993), as documented by the presence of peroxidation products (Young et al. 1995) or enhanced generation of superoxide anions (Chang et al. 1993). Studies from several laboratories indicate that antioxidants improve NO-mediated endothelium-dependent relaxation in vessels from diabetic animals (Diederich et al. 1994, Rodríguez-Mañas et al. 1998, Sena et al. 2008). The main objective of this work was to analyze whether gliclazide ameliorated normal endothelial function in this rodent model of type 2 diabetes with dyslipidemia. Gliclazide has metabolic effects since this drug stimulates insulin secretion, while it also reduces insulin resistance. Therefore, our observed effects can be attributed to both direct vascular actions of gliclazide and improved insulin secretion/function. Oxidative stress and biochemical parameters of the diabetic rats were compared. When diabetic rats were treated with $10 \mathrm{mg} / \mathrm{kg} /$ day gliclazide, a reduction of fasting glycemia and lipid profile to control diabetic levels was observed, while there were no changes in body weight and two-hour postprandial blood glucose. Furthermore, FFAs, 8-OHdG and protein carbonyl groups were significantly reduced after treatment with gliclazide to the same levels as in nondiabetic Wistar rats.
It is interesting to note that the addition of $10 \mu \mathrm{M}$ gliclazide to isolated vessels from GKAD rats caused no effect. This lack of in vitro effect suggests that a direct and immediate free radical scavenging effect of gliclazide in vivo (Scott et al. 1991) cannot account for the improvement in diabetic endothelial cell dysfunction. The effect of gliclazide in vivo could be due to either a chronic increase of endothelium-derived vasodilators or a decrease of vasoconstrictors. In addition, there is a reduction in free radical generation or an increase in free radical scavenging. In diabetic patients treated with gliclazide, plasma reduced thiols were increased, lipid peroxides were decreased, and erythrocyte superoxide dismutase activity was elevated (Jennings et al. 1992). Our results are also consistent with the idea that gliclazide improves diabetic endothelial dysfunction by an antioxidant mechanism (Jennings et al. 1992, Desfaits et al. 1997, O'Brien and Luo 1997). However, we cannot rule out that the metabolic effects of gliclazide may also contribute to these observations. It is possible that the effect of gliclazide was at least partly mediated via improved insulin action as evidenced by the observed decrease in blood glucose levels and insulin resistance. In agreement with our results of improved endothelial function, Pagano et al. (1998) reported that treatment with gliclazide enhances ACh-induced relaxation in isolated aortic segments of alloxan-induced diabetic rabbits.

In addition, the improvement in endothelial function has been reported in streptozotocin-induced diabetic rats receiving gliclazide (Vallejo et al. 2000a). Others have also reported that gliclazide has antioxidant properties in vitro, perhaps reversing the endothelial dysfunction caused by highly glycosylated oxyhemoglobin in human mesenteric microvessels 
(Vallejo et al. 2000b).

The major limitation of this study is that gliclazide decreased fasting blood glucose thus we cannot rule out that gliclazide leads to improvement of oxidative stress and endothelial dysfunction due to the improvement in fasting glucose levels.

A main conclusion of the present study is that gliclazide ameliorates the endothelial dysfunction present in diabetic rats fed a high-fat diet. These beneficial effects on the vasculature may be related to the metabolic actions of the drug, to the improvements in plasma lipids and fasting glycemia and to its antioxidant properties.
Indeed, this work shows that gliclazide is able to reduce fasting hyperglycemia and systemic oxidative stress, thus contributing to increased sensitivity to NO-mediated vasodilation in diabetic GK rats with dyslipidemia.

\section{Conflict of Interest}

There is no conflict of interest.

\section{Acknowledgements}

We wish to thank Laboratory of Experimental research for animal care. This work was supported by grants from Faculty of Medicine of Coimbra and III.

\section{References}

BAYNES JW: Role of oxidative stress in development of complications in diabetes. Diabetes 40: 405-412, 1991.

CAMPBELL DB, LAVIELLE R, NATHAN C: The mode of action and clinical pharmacology of gliclazide: a review. Diabetes Res Clin Pract 14 (Suppl 2): S21-S36, 1991.

CERIELlO A, QUATRARO A, GIUGLIANO D: Diabetes mellitus and hypertension: the possible role of hyperglycaemia through oxidative stress. Diabetologia 36: 265-266, 1993.

CHANG KC, CHUNG SY, CHONG WS, SUH JS, KIM SH, NOH HW, SEONG BW, KO HJ, CHUN KW: Possible superoxide radical-induced alteration of vascular reactivity in aortas from streptozotocin- treated rats. J Pharmacol Exp Ther 266: 992-1000, 1993.

CHENG ZJ, VASKONEN T, TIKKANEN I, NURMINEN K, RUSKOAHO H, VAPAATALO H, MULLER D, PARK JK, LUFT FC, MERVAALA EM: Endothelial dysfunction and salt-sensitive hypertension in spontaneously diabetic Goto-Kakizaki rats. Hypertension 37: 433-439, 2001.

DA ROS R, ASSALONI R, CERIELLO A: Antioxidant therapy in diabetic complications: what is new? Curr Vasc Pharmacol 2: 335-341, 2004.

DESFAITS AC, SERRI O, RENIER G: Gliclazide decreases cell mediated low-density lipoprotein (LDL) oxidation and reduces monocyte adhesion to endothelial cells induced by oxidatively modified LDL. Metabolism 46: 1150-1156, 1997.

DIEDERICH D, SKOPEC J, DIEDERICH A, DAI FX: Endothelial dysfunction in mesenteric resistance arteries of diabetic rats: role of free radicals. Am J Physiol 266: H1153-H1161, 1994.

DURANTE W, SEN AK, SUNAHARA FA: Impairment of endothelium- dependent relaxation in aortae from spontaneously diabetic rats. Br J Pharmacol 94: 463-468, 1988.

FORTES ZB, LEME JG, SCIVOLETTO R: Vascular reactivity in diabetes mellitus: role of the endothelial cells. $\mathrm{Br} J$ Pharmacol 79: 771-781, 1983.

GIUGLIANO D, CERIELLO A, PAOLISSO G: Oxidative stress and diabetic vascular complications. Diabetes Care 19: 257-267, 1996.

HAMILTON SJ, CHEW GT, WATTS GF: Therapeutic regulation of endothelial dysfunction in type 2 diabetes mellitus. Diabetes Vasc Dis Res 4: 89-102, 2007.

JENNINGS PE, SCOTT NA, SANIABADI AR, BELCH JJF: Effects of gliclazide on platelet reactivity and free radicals in type II diabetic patients: clinical assessments. Metabolism 41: 36-39, 1992.

NICHOLS CG, LEDERER WJ: Adenosine triphosphate sensitive potassium channels in the cardiovascular system. Am J Physiol 261: H1675-H1686, 1992.

O'BRIEN RC, LUO M: The effects of gliclazide and other sulfonylureas on low-density lipoprotein oxidation in vitro. Metabolism 46: 22-25, 1997.

O'BRIEN RC, LUO M, BALAZS N, MERCURI J: In vitro and in vivo antioxidant properties of gliclazide. J DiabetesComplications 14: 201-206, 2000. 
PAGANO PJ, GRISWOLD MC, RAVEL D, COHEN RA: Vascular action of the hypoglycaemic agent gliclazide in diabetic rabbits. Diabetologia 41: 9-15, 1998.

PALMER KJ, BROGDEN RN: Gliclazide. An update of its pharmacological properties and therapeutic efficacy in noninsulin dependent diabetes mellitus. Drugs 46: 92-125, 1993.

PENNATHUR S, HEINECKE JW: Mechanisms of oxidative stress in diabetes: implications for the pathogenesis of vascular disease and antioxidant therapy. Front Biosci 9: 565-574, 2004.

RODRÍGUEZ-MAÑAS L, ANGULO J, PEIRÓ C, LLERGO JL, SÁNCHEZ-FERRER P, LÓPEZ-DÓRIGA P, SÁNCHEZ-FERRER CF: Endothelial dysfunction and metabolic control in streptozotocin-induced diabetic rats. Br J Pharmacol 123: 1495-1502, 1998.

SCOTT NA, JENNINGS PE, BROWN J, BELCH JJF: Gliclazide: a free radical scavenger. Eur J Pharmacol 208: 175177, 1991.

SEIÇA RM, SUZUKI KI, SANTOS RM, ROSÁRIO LM: Impaired insulin secretion in isolated islets of Goto-Kakizaki rats, an animal model of non obese type 2 diabetes, is a primary event. (in Portuguese) Acta Med Port 17: 4248, 2004.

SENA CM, BAROSA C, NUNES E, SEICA R, JONES JG: Sources of endogenous glucose production in the GotoKakizaki diabetic rat. Diabetes Metab 33: 296-302, 2007.

SENA CM, NUNES E, LOURO T, PROENÇA T, FERNANDES R, BOARDER MR, SEIÇA RM: Effects of alphalipoic acid on endothelial function in aged diabetic and high-fat fed rats. Br J Pharmacol 153: 894-906, 2008.

VALLEJO S, ANGULO J, PEIRÓ C, NAVADO J, SÁNCHEZ-FERRER A, PETIDIER R, SÁNCHEZ-FERRER CF, RODRÍGUEZ-MAÑAS L: Highly glycated oxyhaemoglobin impairs nitric oxide relaxations in human mesenteric microvessels. Diabetologia 43: 83-90, 2000b.

VALLEJO S, ANGULO J, PEIRÓ C, SÁNCHEZ-FERRER A, CERCAS E, LLERGO JL, NEVADO J, SÁNCHEZFERRER CF, RODRÍGUEZ-MAÑAS L: Prevention of endothelial dysfunction in streptozotocin-induced diabetic rats by gliclazide treatment. $J$ Diabetes Complications 14: 224-233, 2000a.

YOUNG IS, TATE S, LIGHTBODY JH, MCMASTER D, TRIMBLE ER: The effects of desferoxamine and ascorbate on oxidative stress in the streptozotocin diabetic rat. Free Radical Biol Med 18: 833-840, 1995. 